The Astrophysical Journal, 682:1087-1094, 2008 August 1

(C) 2008. The American Astronomical Society. All rights reserved. Printed in U.S.A.

\title{
A LARGE INFRARED SHELL ASSOCIATED WITH BI CRUCIS
}

\author{
Bruce McCollum \\ Spitzer Science Center, California Institute of Technology, MC 314-6, \\ Pasadena, CA 91125; mccollum@ipac.caltech.edu \\ Frederick C. Bruhweiler and Glenn M. Wahlgren \\ Institute for Astrophysics and Computational Sciences, Catholic University of America, \\ Washington, DC 20064; bruhweiler@cua.edu, glenn.m.wahlgren@nasa.gov \\ Mattias ERIKSSON \\ University of Kalmar, 39182 Kalmar, Sweden; mattias.eriksson@hik.se \\ AND \\ EKATERINA VERNER \\ Institute for Astrophysics and Computational Sciences, Catholic University of America, \\ Washington, DC 20064; kverner@milkyway.gsfc.nasa.gov \\ Received 2007 November 26; accepted 2008 April 4
}

\begin{abstract}
Spitzer IRAC and MIPS images reveal a large dust emission feature $\sim 6^{\prime}$ in diameter which appears to be a shell in close proximity to, and perhaps physically related to, the D-type Mira symbiotic BI Cru. Smaller optical lobes are already known to be emanating from some symbiotics including BI Cru. However, this is the first extended structure found in the IR which is associated with a symbiotic Mira system. The IR shell of BI Cru is more than 5 times larger in arc size than the star's optical lobe. Published distance estimates imply that the IR shell is $\sim 4$ to $\sim 8 \mathrm{pc}$ in diameter, which is larger than the largest optical lobe known to be associated with any Mira symbiotic system. The large disparity between its IR and optical shell sizes, along with what appear to be multiple intersecting arcs, suggest that BI Cru has undergone multiple mass-loss episodes. A trend of rapidly increasing brightness toward longer wavelengths, along with a much more diffuse structure at $70 \mu \mathrm{m}$ than at shorter wavelengths, and suggests a greater abundance of relatively colder and older dust which may be the remnant of earlier mass outflows.
\end{abstract}

Subject headings: binaries: symbiotic — ISM: bubbles — stars: individual (BI Crucis) — stars: mass loss stars: winds, outflows

\section{INTRODUCTION}

BI Crucis is a symbiotic star (Henize \& Carlson 1980) with strong $\mathrm{H} \alpha$ emission (Henize 1976), consisting of a Mira variable (Allen 1974) and a hot compact object. Its near-IR emission was interpreted as being from circumstellar dust at $1300 \mathrm{~K}$ (Allen 1974; Allen \& Glass 1975). Rossi et al. (1988) concluded instead that a composite spectrum is needed to fit the optical to mid-IR SED of BI Cru, and classed it as a D-type (dusty) symbiotic. Rossi et al. also found that there is a substantial uncertainty in the amount of circumstellar dust and degree of obscuration of the Mira, and that the dust envelope could be as cool as $200 \mathrm{~K}$.

Schwarz \& Corradi (1992) discovered a large, clumpy, optical bipolar nebula associated with BI Cru which extends to $\sim 80^{\prime \prime}$ from the star in [N II] (Corradi \& Schwarz 1995) and in [O III] (Schwarz \& Corradi 1992). Schwarz \& Corradi (1992) confirmed the presence of a Mira along with an accretion disk around a compact object which they presumed to be a white dwarf.

Optical nebulae around symbiotic Miras are common. A literature survey by Corradi et al. (1999a) found that about $40 \%$ of Mira symbiotics have extended ionized nebulae which range in size from a few arcseconds in diameter to $150^{\prime \prime}$, the largest known so far being that of BI Cru, and which have a variety of morphologies. However, until now no structured, extended IR emission has been detected associated with a symbiotic Mira.

The high spatial resolution and sensitivity of the Spitzer Space Telescope in the mid-IR allowed the detailed imaging of almost 600 partial and closed IR shells in images of the Spitzer Galactic plane surveys GLIMPSE I and GLIMPSE II by Churchwell et al. $(2006,2007)$. Based on our examination of the GLIMPSE archival data, we have discovered that the edge of one such shell spatially overlaps BI Cru. We present reasons why the shell is probably the result of a mass outflow from the BI Cru system.

\section{OBSERVATIONS}

We used Spitzer (Werner 2004) archival data from two Spitzer Legacy surveys: GLIMPSE (Benjamin et al. 2003), which utilized the IRAC camera (Fazio et al. 2004), and MIPSGAL (Carey et al. (2005), which used the MIPS detector (Rieke et al. 2004). The IRAC obtains images at bandpasses centered on 3.6, 4.5, 5.8, and $8.0 \mu \mathrm{m}$ with a field of view of $5.21 \times 5.21 \mathrm{arcmin}^{2}$ in each image. Additional processing was performed by the GLIMPSE team to remove artifacts and to produce final image mosaics with pixel sizes of $0.6 \times 0.6 \operatorname{arcsec}^{2}$. MIPSGAL obtained images at 24 and $70 \mu \mathrm{m}$, with a resolution of $2.55^{\prime \prime}$ pixel $^{-1}$ at $24 \mu \mathrm{m}$ and $\sim 10^{\prime \prime}$ pixel $^{-1}$ at $70 \mu \mathrm{m}$. In addition, we obtained MIPS 24 and $70 \mu \mathrm{m}$ images as part of our own Guest Observer program to study symbiotic stars, which were reduced with the standard Spitzer pipeline.

\section{DISCUSSION}

\subsection{Characteristics of the Extended Emission}

The IRAC and MIPSGAL images reveal arcs of IR emission delineating a slightly ovoidal shell $\sim 6^{\prime}$ in diameter, adjacent to 

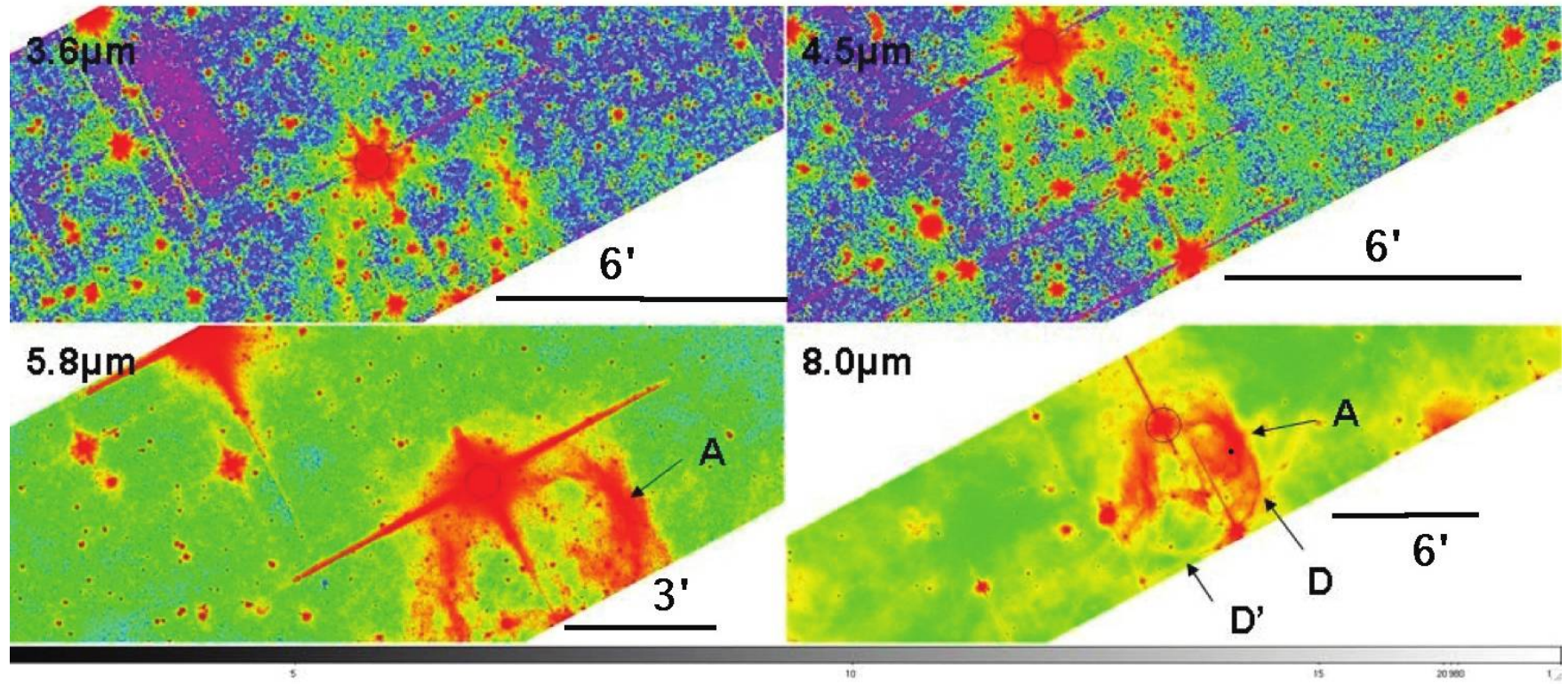

FIG. 1. - IRAC images of BI Cru area, displayed using histogram equalization. Scale is $0.6^{\prime \prime}$ pixel $^{-1}$. North is up, east is left. BI Cru is marked with black circle. Clockwise from upper left, images show emission at 3.6, 4.5, 5.8, and $8 \mu \mathrm{m}$. A, D, and $\mathrm{D}^{\prime}$ show some sections of the outer arc. After estimated local ISM background is subtracted, colors show flux densities in extended emission regions as follows: (a) at $3.6 \mu \mathrm{m}, \sim 1-2 \mathrm{MJy} \mathrm{sr}^{-1}$ (green-yellow) to a maximum of $\sim 3.5 \mathrm{MJy}^{-1}$ (orange); (b) at $4.5 \mu \mathrm{m}, \sim 0.6-1.2 \mathrm{MJy} \mathrm{sr}^{-1} ;(c)$ at $5.8 \mu \mathrm{m}, \sim 4$ (green) to $\sim 8 \mathrm{MJy} \mathrm{sr}^{-1} ;(d)$ at $8 \mu \mathrm{m}, \sim 5$ ( green) to $\sim 8$ (yellow) to $\sim 25 \mathrm{MJy} \mathrm{sr}^{-1}$ (dark orange).

BI Cru, which are defined by the filaments marked A through E in Figures 1 and 2.

The IRAC shell adjacent to BI Cru is listed as S174 in the catalog of Churchwell et al. (2006, 2007). Churchwell et al. (2006) provide a composite four-color IRAC image of the shell, but no MIPSGAL image has been published. Because the emission and apparent extent of the BI Cru shell increases greatly from shorter to longer IRAC wavelengths, the four-color composite image is so dominated by $8 \mu \mathrm{m}$ emission that it reveals little of the structure at shorter wavelengths. In addition, the image of Churchwell et al. (2006) did not have the improved spatial resolution of the
GLIMPSE final archived products. Therefore, in this paper we show the higher resolution archival images, with each wavelength displayed separately, and with the display scales of each image adjusted in order to best reveal the details of the structure. Furthermore, Churchwell et al. $(2006,2007)$ do not discuss this shell individually other than to list its spatial dimensions in their online catalog of shells, so we begin with a detailed discussion of this object.

Based on subjective measurements by four independent examiners, Churchwell et al. (2006) report this shell as having an approximately elliptical shape with an eccentricity of 0.50 , a major

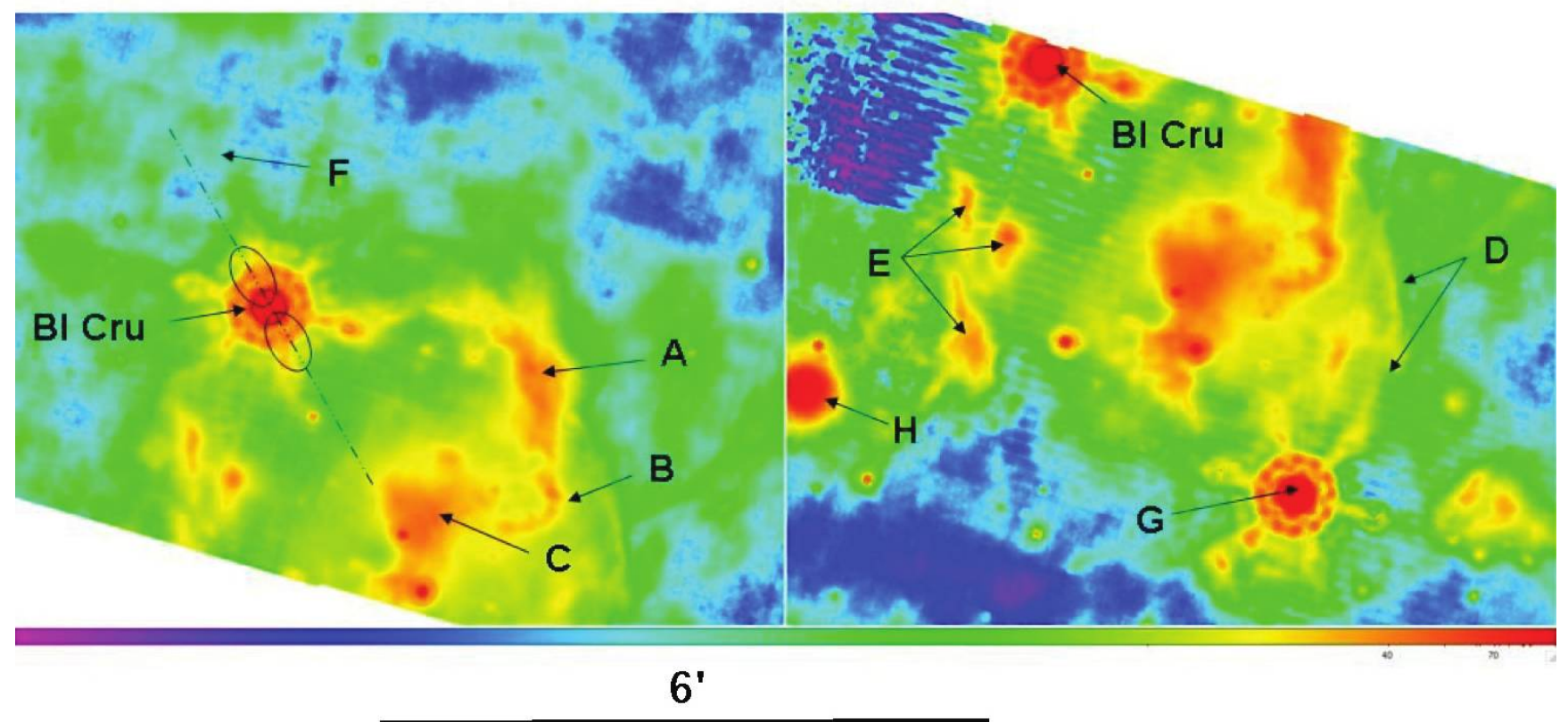

FIG. 2.-BI Cru area at $24 \mu \mathrm{m}$, histogram equalization. Resolution is $2.55^{\prime \prime}$ pixel $^{-1}$. North is up, east is left. Black ovals show size and orientation of optical lobes. $\mathrm{A}, \mathrm{D}$, and $\mathrm{D}^{\prime}$ indicate parts of the shell. F indicates location of some faint extended emission ( green) northeast of BI Cru which is of uncertain nature (ISM or ejecta). Background-subtracted flux densities in extended material in $\mathrm{MJy} \mathrm{sr}^{-1}$ are $\sim 3$ (green), $\sim 7$ (yellow), up to $\sim 20-25$ (red-orange). 


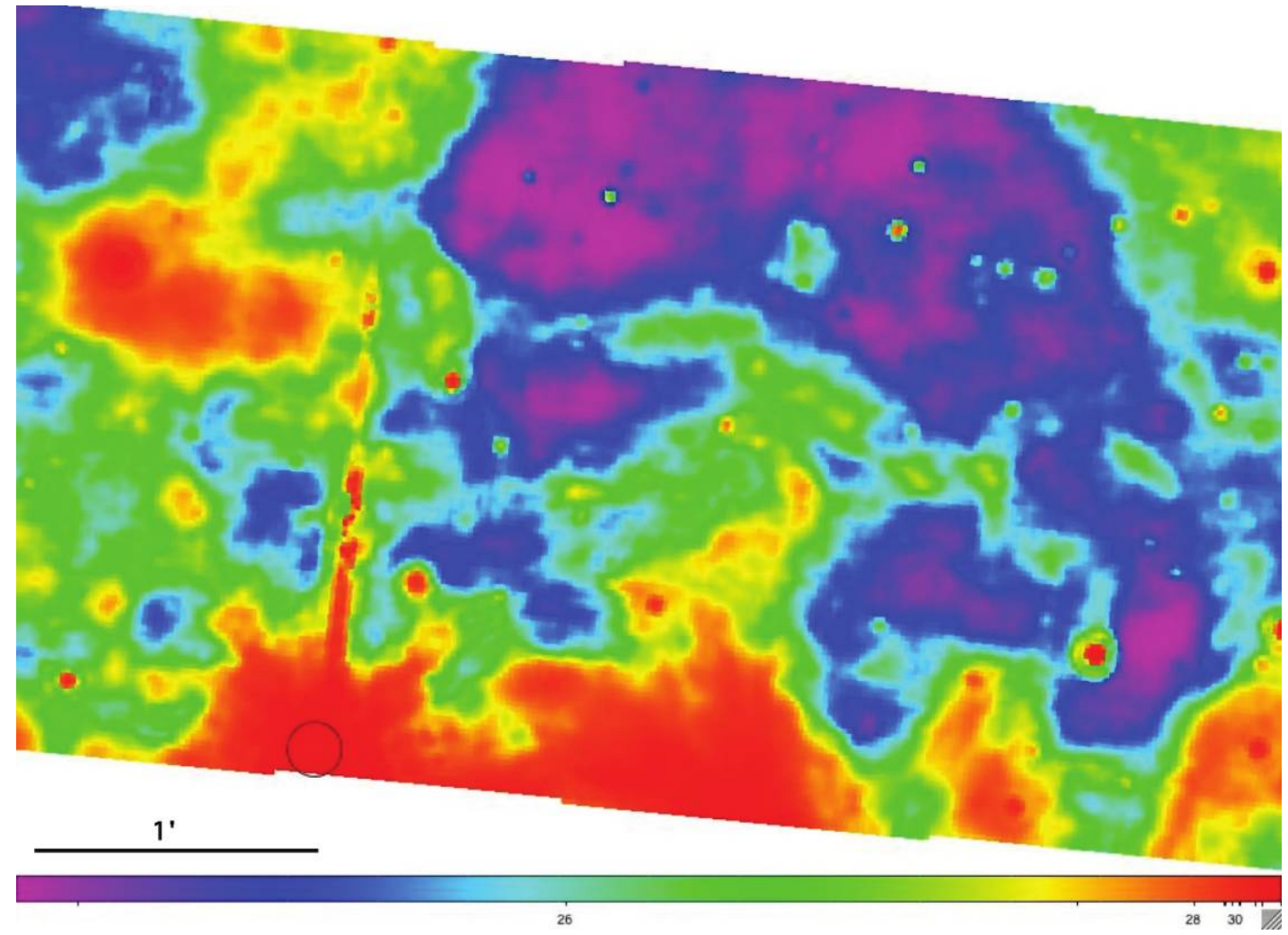

FIG. 3.-MIPS $24 \mu \mathrm{m}$ deep image of north side of BI Cru, histogram equalization. North is up, east is left. Image size is $5.4 \times 2.7 \mathrm{arcmin}^{2}$. Resolution is $2.55^{\prime \prime}$ pixel ${ }^{-1}$. Bright, short radial features extending from BI Cru are artifacts. Background-subtracted flux densities in the extended material range from $\sim 1-2 \mathrm{MJy} \mathrm{sr}^{-1}($ blue and green $)$ to $\sim 3 \mathrm{MJy} \mathrm{sr}^{-1}$ (lighter orange) to $\sim 10-15 \mathrm{MJy} \mathrm{sr}^{-1}$ (brightest parts of red outside of BI Cru PSF).

axis of the outer shell boundary of $6.76^{\prime}$, a minor axis of the outer shell boundary of $5.84^{\prime}$, and an average radius of $2.84^{\prime}$. Although an estimate of the position angles of the shell axes is not provided, a visual examination of the Churchwell et al. images suggests that the major axis is oriented approximately along a line through BI Cru.

In IRAC images (Fig. 1), relatively little extended emission is apparent at 3.6 and $4.5 \mu \mathrm{m}$. That present is mostly in the form of a faint ring passing across BI Cru. In the 5 and $8 \mu \mathrm{m}$ bands, complex IR-emitting structures are seen including multiple intersecting arcs. The IRAC image resolution is about 4 times higher than that at $24 \mu \mathrm{m}$ and about 16 times higher than that at $70 \mu \mathrm{m}$, but a roughly similar structure is seen in both MIPS and IRAC images. The longer wavelength emission is more irregular, but its outer boundaries rather closely match the outer boundaries of the shell which is delineated in the IRAC images. The location to the southwest of BI Cru is consistent with the axis of the smallscale optical bipolar ejecta at P.A $=-149^{\circ}$ shown by Schwarz $\&$ Corradi (1992). A comparison of optical and IR features within $10^{\prime \prime}$ of BI Cru is not possible because Schwarz \& Corradi (1992) used a $20^{\prime \prime}$ occulting disk to cut out light contamination from BI Cru. Some fainter, more diffuse emission is seen north and east of BI Cru at $8 \mu \mathrm{m}$, but any IRAC images near the Galactic plane are typically heavily contaminated by emission from interstellar clouds (Benjamin et al. 2003), so the faint emission northward, because it is not clearly structured or strikingly brighter than the ISM nearby, cannot confidently be attributed to something besides the ISM.

The $70 \mu \mathrm{m}$ flux densities of the extended material are mostly a few to several times greater than the $24 \mu \mathrm{m}$ flux densities, suggesting that there is larger quantity of colder and presumably older material. Flux densities of the brightest parts of the ma- terial increase from a few $\mathrm{MJy} \mathrm{sr}^{-1}$ at IRAC wavelengths to over $200 \mathrm{MJy} \mathrm{sr}^{-1}$ at $70 \mu \mathrm{m}$ after rough estimates of the complex ISM background are subtracted. The extended material is typically a factor of a few brighter than the nearby ISM at all wavelengths. (We emphasize that the ISM background at Spitzer wavelengths is not Poisson noise. The per-pixel $\mathrm{S} / \mathrm{N}$ of these images is typically several tens to 1 , depending on wavelength [Benjamin et al. 2003; Carey et al. 2005]). Because the spatially complex ISM background cannot be distinguished from IR-shell emission where the two sources overlap, our estimated IR-shell flux densities must be considered only approximate upper limits to the shell emission.

The MIPSGAL $24 \mu \mathrm{m}$ images (Fig. 2) show emission that is clumpier and more diffuse than at the IRAC wavelengths, but that still matches the overall spatial extent and location of the IRAC features. At $24 \mu \mathrm{m}$ there are some barely visible faint arcs, indicated by $\mathrm{F}$ in Figure 2, but these features are so small and faint that we are uncertain whether or not they are unrelated background features. As part of our own Spitzer program to observe symbiotic stars and search for smaller scale ejecta from symbiotic stars, we obtained a deeper $24 \mu \mathrm{m}$ exposure (3 times longer per-pixel integration time; Fig. 3) north of BI Cru, but that image, too, revealed no extended emission clearly associated with BI Cru. The $70 \mu \mathrm{m}$ MIPSGAL images (Fig. 4) show emission which is both brighter and more diffuse than at shorter wavelengths but overall matches well the spatial location and extent of that seen at shorter wavelengths. We obtained a 3 times deeper exposure at $70 \mu \mathrm{m}$ immediately north of BI Cru, where the MIPSGAL image shows no extended emission associated with the star (Fig. 5). This additional image, too, did not reveal any extended emission with structure, apart from part of the arc west of the star which was already seen in MIPSGAL images. The scalloped shape on the interior side of filament B implies that lower density material is moving 


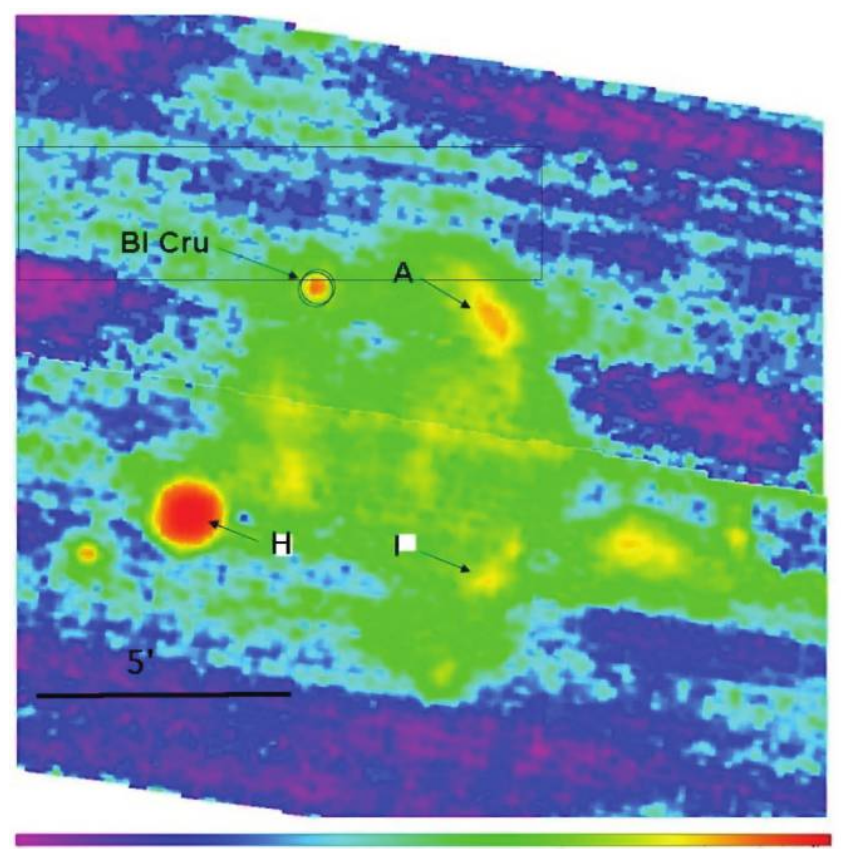

FIG. 4.-MIPSGAL $70 \mu \mathrm{m}$ images of BI Cru area, histogram equalization. North is up, east is left. Resolution is $5^{\prime \prime}$ pixel $^{-1}$. Position of BI Cru is marked with black circles. A and I indicate bright sections of the shell. Backgroundsubtracted flux density brightness scale in $\mathrm{MJy} \mathrm{sr}^{-1}$ is $\sim 60$ (green), $\sim 130$ (yellow), $\sim 160$ (orange), up to a maximum of $\sim 230 \mathrm{MJy} \mathrm{sr}^{-1}$. Light blue horizontal striations are artifacts.

into a denser region to the southwest, and possibly around a dense condensation (C).

Churchwell et al. $(2006,2007)$ state that the $24 \mu \mathrm{m}$ emission in their images always appears to be bounded by the $8 \mu \mathrm{m}$ shell, which we find is also the case for the BI Cru shell. They also comment that within some of the shells, the $24 \mu \mathrm{m}$ emission has a peak near the center of shell, which is similar to a feature of the BI Cru shell (C in Fig. 2). They consider the existence of a center peak at $24 \mu \mathrm{m}$ is difficult to explain, for various reasons. They do not discuss $70 \mu \mathrm{m}$ emission associated with any shells, give IRAC shell fluxes, or discuss the IR SEDs of the shells except to state that the shells are usually seen in all IRAC wave bands and "tend to be brighter at longer wavelengths."

Although we refer to the overall BI Cru extended emission as a shell, it is seen that the emission becomes much more diffuse at $24 \mu \mathrm{m}$ and especially $70 \mu \mathrm{m}$ although it remains within the boundaries outlined by the short-wavelength shell. The fact that the interior of the shell tends to be filled in with substantial emission at 24 and $70 \mu \mathrm{m}$ is difficult to reconcile with a simple shelllike distribution of dust. Indeed, if only 24 and $70 \mu \mathrm{m}$ images were available, we probably instead would have chosen to refer to this object as a fairly filled-in lobe.

\subsection{Comparison with the Dimensions of Other Shells}

Churchwell et al. $(2006,2007)$ present little information about most shells except for a variety of morphological statistics, which they cross-compare in histogram form. Churchwell et al. (2006) caution that, for a variety of reasons, their catalog of shells is perhaps only about $50 \%$ complete even within the limited coverage of the survey, and has selection biases. Furthermore, they report that the shell thicknesses estimated by their examiners had a dispersion of $\sim 10 \%$, and the estimated thicknesses and eccentricities varied by $\sim 35 \%$.

The dimensions of the BI Cru shell are slightly atypical for shells measured by Churchwell et al. (2006, 2007). The average angular diameter of 5.36' estimated by Churchwell et al. (2006) for the BI Cru shell puts it among the largest 20 out of 322 shells. (The largest in their list is $\sim 25^{\prime}$ in diameter.) While its average estimated angular thickness of $0.6^{\prime}$ is about average, it is among the thinnest $\sim 20 \%$ in that the ratio of its average thickness to average radius is 0.21 . Its estimated eccentricity places it in the least eccentric $\sim 20 \%$ as well. Thus the BI Cru shell is among the few largest, roundest, and thinnest of those in the Churchwell et al. (2006) catalog. Based on the discussions of Churchwell et al. (2006, 2007) regarding those statistics, little can be concluded. However, Churchwell et al. (2006) note that according to the theory

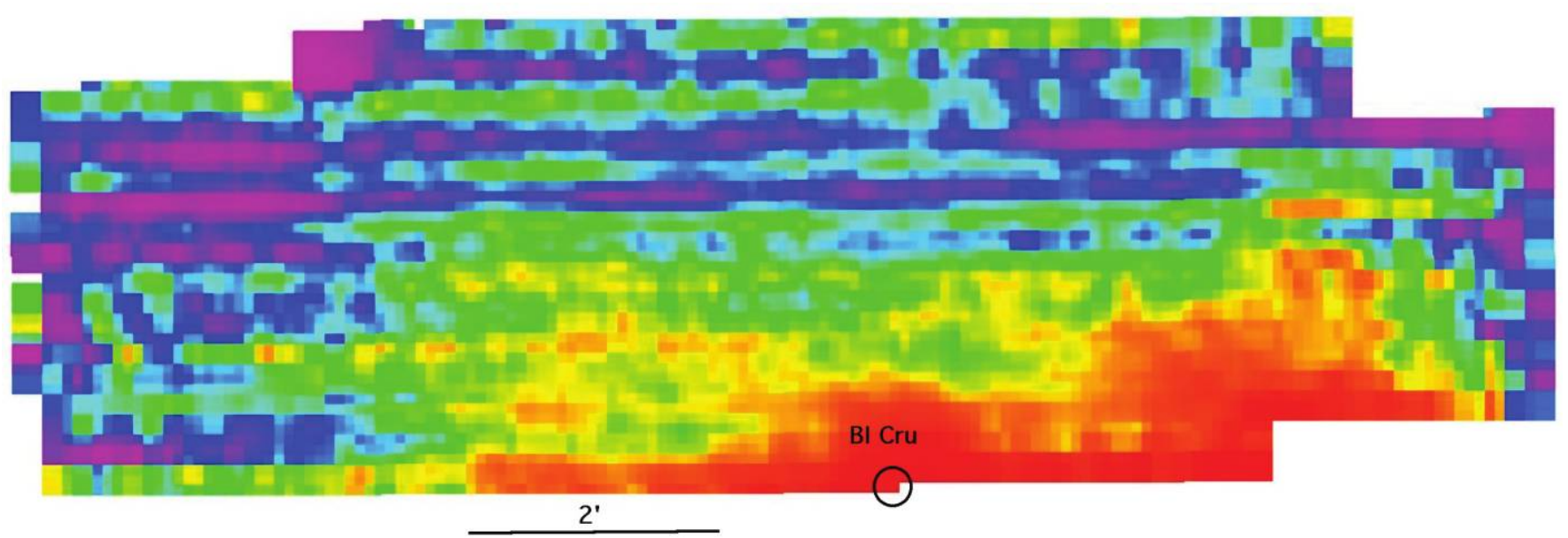

100

FIG. 5.- MIPS $70 \mu \mathrm{m}$ deep exposure of north side of BI Cru, histogram equalization. North is up, east is left. Resolution is $2.55^{\prime \prime}$ pixel ${ }^{-1}$. Image size is $5.25 \times 2.6$ arcmin $^{2}$.

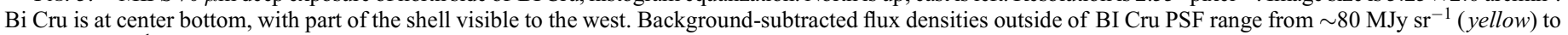
$\sim 220 \mathrm{MJy} \mathrm{sr}^{-1}$ (red-orange). 
of Weaver et al. (1977), the shell thickness of a dynamically blown bubble from a single early-type central star tends to increase linearly with shell radius, and the ratio of thickness to radius should increase with age and with the density of the ambient ISM. We speculate, therefore, that the small thickness-to-radius ratio of the BI Cru shell might either indicate a relatively young shell age, or a relatively dense ambient ISM, which would be expected if the shell material is an outflow from BI Cru into preexisting, older material from previous mass loss episodes of that system.

\subsection{Possible Sources of the Ejecta}

Churchwell et al. $(2006,2007)$ find evidence that only a very few shells are produced by supernova remnants (SNRs) or planetary nebulae (PNe). They argue that most of the shells are produced by $\sim \mathrm{B} 4$ and later dwarfs which cannot create detectable $\mathrm{H}$ II regions but can still create dust bubbles with radiation pressure. They admit, however, that many bubbles do not have good candidate central stars or star clusters. They do not mention considering symbiotic stars as a possible source of any of the shells. Although they do not discuss the BI Cru shell specifically or note that it is adjacent to BI Cru, in their catalog they remark that this shell does not have a candidate central star or any other apparent source of origin, and therefore consider the shell's origin to be unknown.

Churchwell et al. $(2006,2007)$ do not discuss whether the shell might have originated from a bright object on the shell itself, so we next consider that possibility in more detail. The 2MASS Catalog (Skrutskie 2006) shows well over 100 2MASS point sources within the entire IRAC $3.6 \mu \mathrm{m}$ FOV, 20 of those being within $\sim 30^{\prime \prime}$ of IRAS 12201-6226 alone, for example. Some 2MASS sources coincide spatially with the IR structure, but the density of 2MASS sources makes some chance superpositions likely. However, we investigated the literature for information on the nature of three notably bright objects which are found in or near parts of the outer boundary of the shell and which match the coordinates of IRAS PSC sources.

The brightest arc section (filament A in Fig. 1) is about $30^{\prime \prime}$ west of BI Cru. Part of the inner edge of this feature falls within the position uncertainty ellipse $\left(6 \times 11 \operatorname{arcsec}^{2}\right)$ of IRAS $12202-$ 6222 , for which no literature references exist. The uncertainty ellipse of this IRAS source is marked with a dark ellipse in the $8 \mu \mathrm{m}$ image in Figure 1. The CDS VizieR shows no optical or IR object within $13^{\prime \prime}$ of the IRAS position. The Spitzer IRAC and MIPS images also reveal no point sources within $13^{\prime \prime}$ of IRAS 12202-6222. In the IRAC and $24 \mu \mathrm{m}$ images, filament $\mathrm{A}$ has the appearance of two arcs intersecting at different angles, one being an extension of $\mathrm{D}$ and the other being from the brighter periphery of the shell outlined by A, B, C, and E in Figure 2. The IRAS fluxes of the 12202-6222 at 12, 25, 60, and $100 \mu \mathrm{m}$ are approximately $0.07,1.0,21$, and $94 \mathrm{Jy}$, respectively, which are approximately consistent with Spitzer fluxes. We therefore conclude that IRAS $12202-6222$ is not a point source, but, instead, is filament A.

IRAS 12210-6225 appears as a bright, circular, extended object near the arc southeast of BI Cru. It is feature H in Figures 2 and 4. It has no references in the literature. Its diameter is $\sim 40^{\prime \prime}$ at $8 \mu \mathrm{m}$ and $\sim 30^{\prime \prime}$ in the three shorter IRAC bandpasses. Its IRAS positional uncertainty ellipse is $3 \times 11 \operatorname{arcsec}^{2}$, with an ellipse orientation uncertainty of $142^{\circ}$. Several unstudied optical and near-IR point sources overlap with the diffuse $8 \mu \mathrm{m}$ object. The 2MASS Catalog lists five sources with distances from IRAS 12210-6225 ranging from $\sim 4^{\prime \prime}-10^{\prime \prime}$, with $K$ magnitudes from 12.7 to 14.2. The USNO-B1.0 Catalog (Monet et al. 2003) lists 13 objects with distances from $6^{\prime \prime}$ to $18^{\prime \prime}$ from the IRAS coordi- nates, which have $B$ magnitudes from 18.0 to 19.4 and $R$ magnitudes from $\sim 14$ to 19 , which have no published information except catalog entries. There is no reason to think that any of them are physically related to IRAS $12210-6225$. Their faintness relative to $\mathrm{BI} \mathrm{Cru}(B \sim 12, K \sim 5)$ tends to argue against any of them being the source of the arc. Also, the fact that IRAS 12210-6225 does not connect as well with the arc, especially in MIPS images, tends to argue against it being a source of the material.

IRAS $12201-6226$ (G in Fig. 2) is an IRAS point source with an uncertainty ellipse of $4 \times 13 \operatorname{arcsec}^{2}$. A catalog search using the CDS VizieR found no optical sources within $4^{\prime \prime}$ of the apparent center of the IRAC source. The 2MASS Catalog lists an object at $12^{\mathrm{h}} 22^{\mathrm{m}} 55^{\mathrm{s}}(\mathrm{J} 2000.0),-62^{\circ} 43^{\prime} 13^{\prime \prime}$, at about the apparent center of IRAC object $\mathrm{G}$, having $J, H$, and $K$ magnitudes of about $12.4,10,4$, and 8.4 , respectively, but its $B$ and $V$ magnitudes have an upper limit of 22. ${ }^{1}$ Finlator et al. (2000) found that it is not possible to perform reliable spectral classification using 2MASS colors alone, but that stars may be sorted into broad groups of spectral types using 2MASS color-color plots. The 2MASS colors of IRAS 12201-6226 are redder than those of any objects including M stars plotted by Finlator et al. (2000), and redder than the T dwarfs examined by Burgasser et al. (2003). It is not visible as a point source at $70 \mu \mathrm{m}$, but its position coincides with an irregular bright feature at that wavelength (I in Fig. 4).We conclude that IRAS $12201-6226$ is probably a background object which is highly reddened by a relatively dense patch of dust in the shell.

The IR-shell somewhat resembles that of some SNRs imaged by Spitzer (Reach et al. 2006; Morris et al. 2006), but no SNRs have been identified in the area. The CDS VizieR lists only one radio source overlying the shell, J1223-6238, which does not have a published classification. J1223-6238, was detected at 4.85 GHz as a possible extended source by Wright et al. (1994), centered $1.77^{\prime}$ southwest of BI Cru, having a flux density of $294 \pm 17 \mathrm{mJy}$. BI Cru itself was detected at 8.9 and $14.5 \mathrm{GHz}$ with fluxes of 40.0 and $25.0 \mathrm{mJy}$, respectively (Wendker 1995). We calculated the spectral index $\alpha$, which for SNRs is typically $\alpha \sim 0.5-1.0$ (e.g., Lerche 1980), of the radio source. Its 4.85/ $8.9 \mathrm{GHz}$ fluxes give $\alpha=+0.30$. However, Wright et al. (1994) used a FWHM Gaussian beam width with a semimajor axis of $8.19 \times 3.78$ arcmin $^{2}$, which would have included BI Cru, possibly affecting our estimate of the $4.85 / 8.9 \mathrm{GHz}$ index. Positive spectral indices are consistent with stellar emission but not with nonthermal emission, although many SNRs do not exhibit nonthermal radio emission. The fluxes at $8.9 \mathrm{GHz}$ and $14.5 \mathrm{GHz}$ give an index of +1.04 . Thus the available spectral indices of J12236238 are consistent with those of SNRs, although it has not been identified as an SNR in such surveys. Churchwell et al. (2006) found that only three of their 322 cataloged shells were probable SNRs, so such an origin is statistically improbable in any case.

Churchwell et al. $(2006,2007)$ find, on average, $\sim 1.5$ shells per square degree in the region covered by the GLIMPSE I survey $\left(|l|=10^{\circ}-65^{\circ}\right.$ and $\left.|b| \leq 1^{\circ}\right)$. Most of their identifications of the origins of specific shells rest on the argument that, given the typical diameter of a shell (a few arcminutes), a chance alignment between two uncommon sorts of objects (e.g., shell $+\mathrm{H}$ II region) is highly improbable. They argue that, therefore, if an object which can reasonably be expected to create such a shell is located coincident with the shell, then the two are probably physically related. The GLIMPSE survey covered $\sim 220 \mathrm{deg}^{-2}$ of the Galactic plane. Given that there are only a few hundred symbiotics

\footnotetext{
${ }^{1}$ See N. Zacharias et al. 2005, NOMAD Catalog, at VizieR On-line Data Catalog: I/297.
} 
known in the entire sky, it is highly improbable that BI Cru would be aligned an IR shell by chance, even if there were as many known Mira symbiotics as IR shells, since a region $7^{\prime}$ in diameter would occupy about 155 square minutes of the 3600 square minutes in that square degree. It is still less probable that a shell edge would by chance align with a Mira symbiotic. It is also highly improbable that two unrelated objects (BI Cru and a different object which is the true mass ejector) would both be coincident with the edge of the same shell, so on statistical grounds alone we do not think that any of the other bright IR objects on the BI Cru shell boundary are the source of the shell.

\subsection{Age and Size of the Shell}

Rossi et al. (1988), using IRAS fluxes, estimated the distance to $\mathrm{BI}$ Cru to be $\sim 2000$ pc. However, the bright extended emission we have found near the star at scales much smaller than the $I R A S$ resolution implies that there may have been a large nonstellar contribution to flux in the IRAS $12 \mu \mathrm{m}$ bandpass $(\sim 8-$ $15 \mu \mathrm{m}$; Neugebauer et al. (1984). This would not have been properly taken into account in the distance scaling from IR brightness or in the IR SED modeling of the system. Whitelock et al. (1983) estimated its distance to be $\sim 4400 \mathrm{pc}$ using the Mira period-luminosity relationship, which may be a more accurate distance value because the IR luminosity used would not have been affected by unresolved emission from the shell. A distance of $4400 \mathrm{pc}$ would imply that the BI Cru IR arc is $\sim 8 \mathrm{pc}$ in diameter. Although the shell discussed in this paper is a dust feature, we note for comparison that the largest optical ionized nebula known around a symbiotic is $\sim 1$ pc in diameter.

Although bipolar PNe are different in origin from the observationally similar bipolar nebulae associated with symbiotic systems (e.g., Corradi et al. 1999a, 1999b), the possible connections between bipolar PNe and symbiotics are still being studied (e.g., Balick 2003). However, we note that the estimated optical diameters of 43 known bipolar PNe are in the range of $\sim 0.5-1.8 \mathrm{pc}$ (Corradi \& Schwarz (1995). Corradi \& Schwarz (1995) attributed the large sizes of the PNe to the high expansion velocities observed in those systems.

Optical images (e.g., Schwarz \& Corradi 1992) show large, clumpy emission condensations at several wavelengths, where radial velocities from nebular emission indicate rapid expansion, $v_{\exp } \sim-210 \mathrm{~km} \mathrm{~s}^{-1}$. Henize \& Carlson (1980) found that the BI Cru optical ejecta have a velocity of $\sim 461 \mathrm{~km} \mathrm{~s}^{-1}$. These are among the highest known expansion velocities of such nebulae (Corradi 1993). If we assume the expansion velocity of the IR shell has consistently been $\sim 461 \mathrm{~km} \mathrm{~s}^{-1}$, then we would infer a lower limit to its age of $t_{\mathrm{dyn}} \geq 15 \times 10^{3} \mathrm{yr}$. In comparison, Corradi et al. (1999b) estimate the age of the optical nebula of BI Cru to be 2000 yr.

However, any assumed expansion velocity has substantial uncertainties, as a large range of velocities are usually seen in optical nebulae with such objects, ranging from the typical wind of a red giant $\left(10 \mathrm{~km} \mathrm{~s}^{-1}\right)$, to the many hundreds $\mathrm{km} \mathrm{s}^{-1}$ of the tenuous winds from white dwarfs. Several symbiotics have been found to have jets having velocities of hundreds of $\mathrm{km} \mathrm{s}^{-1}$ to well over $1000 \mathrm{~km} \mathrm{~s}^{-1}$, (Brocksopp et al. 2004 and references therein). Any expansion velocity between these values might be assumed, as source of the IR shell and its dynamical evolution are not understood. Our estimated lower limit of the age of the IR shell assumes that the region interior to the shell was already empty of gas and dust so that the observed IR shell material has not been slowed by interactions. In reality, the region through which the shell has passed might already have contained some material from previous outbursts, or instead might have been swept clear of material because of a previous mass ejection. In general, the cause of outbursts in classical symbiotics is not well understood. It is not known whether all outbursts from the same object should have the same initial velocity, whether high-velocity jets result in significant mass loss, how much mass loss results from symbiotic mass outflows over the lifetime of a system, or whether they all at some point produce large extended structures.

For the 74 shells in the GLIMPSE I survey for which distances could be estimated, Churchwell et al. (2006) found diameters ranging from $\sim 0.1$ to $33.8 \mathrm{pc}$, with a mean of about $6.6 \mathrm{pc}$. Thus, if BI $\mathrm{Cru}$ is assumed to be the origin of the shell, the shell diameter implied by the distance of BI Cru is not anomalous compared to the diameters of the other GLIMPSE I shells.

\subsection{Possible Implications for the Nature of BI Cru}

The source of emission in the IRAC 5.8 and $8.0 \mu \mathrm{m}$ bands, where the shell is brightest, is presumably from polycyclic aromatic hydrocarbons (PAHs), which have strong emission features in those bands. While Churchwell et al. (2007) suggested that in most shells of their catalog, PAH emission was excited by a hot B star, it has also been shown that stars as cool as $3000 \mathrm{~K}$ can produce observable PAH emission in those bands ( $\mathrm{Li} \&$ Draine 2002). Churchwell et al. $(2006,2007)$ postulated for their shells that the longer wavelength emission is thermal emission from dust, which would also be a reasonable presumption if the emission in the shell is the result of one or more mass outflows from BI Cru.

Schwarz \& Corradi (1992) and Corradi \& Schwarz (1993) concluded that the BI Cru system contains both a pre and a postplanetary nebula, and suggested that a faint remnant planetary nebula surrounding BI Cru might be observable although it had not yet been detected, so we discuss here whether the IR shell might be a relic PNe. An argument against the shell being a relic PNe from the hot companion was given by Corradi et al. (1999a), who pointed out that for a very early post-AGB phase (Mira) and a very old post-AGB phase ( $\mathrm{PNe}$ ) to coexist in the same system, because of the short lifetimes of both phases the two stars would need to have had initial masses less than $0.2 \%$ of each other. However, their mass-difference estimate is based on the lifetime of the optical PNe of a WD. A large dust feature, such as we have found with BI Cru, could be older and longer lived than typical $\mathrm{PNe}$, so it could be less unlikely to find a very old relic PN and a Mira in the same system than Corradi et al. (1999a) estimated. Indeed, Corradi et al. (1999a) stated that the same statistical argument of improbably small initial mass differences implies that the hot component of a symbiotic should not be just a typical post-AGB star passing through its hot phase, but rather be an older object which has remained in a hot and luminous phase for an extended time because of mass accretion. Corradi et al. (1999b) speculated that symbiotic Mira systems may produce extended nebulae recurrently every several thousand years.

In this context it is worth noting that the IRAS PSC fluxes for two catalog "point sources" coinciding with parts of the shell distinct from BI Cru have peak fluxes at $100 \mu \mathrm{m}$. BI Cru PSC fluxes decline from $17 \mathrm{Jy}$ at $12 \mu \mathrm{m}$ to $15 \mathrm{Jy}$ at $25 \mu \mathrm{m}$ to a limit of $12 \mathrm{Jy}$ at $60 \mu \mathrm{m}$. In contrast, IRAS 12201-6226 has slightly rising fluxes of $5.4 \mathrm{Jy}$ at $12 \mu \mathrm{m}$ and $8.5 \mathrm{Jy}$ at $25 \mu \mathrm{m}$, with upper limits of $9.9 \mathrm{Jy}$ at $60 \mu \mathrm{m}$ and $120 \mathrm{Jy}$ at $100 \mu \mathrm{m}$. IRAS $12,210-$ 6225 has IRAS fluxes of about $2 \mathrm{Jy}, 5 \mathrm{Jy}, 67 \mathrm{Jy}$, and $119 \mathrm{Jy}$ at 12 , 25,60 , and $100 \mu \mathrm{m}$, respectively. As previously stated, the IRAS fluxes of 12,201-6222, which is nothing more than a bright section of the extended material, increase from less than $1 \mathrm{Jy}$ at $12 \mu \mathrm{m}$ 
to $94 \mathrm{Jy}$ at $100 \mu \mathrm{m}$. An IR SED that rises longward as far as $100 \mu \mathrm{m}$ and perhaps beyond suggests that even colder, older dust may be present in this shell than Spitzer has detected, which might be expected if a reservoir of dust has accumulated in the area from a series of past mass loss episodes. The fact that the extended emission has a much more uniform and diffuse appearance in the Spitzer $70 \mu \mathrm{m}$ images than at shorter wavelengths is also consistent with what one might expect to see from the remnants of older mass outlfows. It might be objected that the current shell might be expected to have swept out a cavity in which there is little dust. However, without knowledge of the three-dimensional distribution of the dust one cannot exclude the possibility that the long-wavelength dust emission is from material outside the short-wavelength shell along the line of sight.

Thus, the dust shell of BI Cru might be a relic from an older mass loss episode than the one currently visible optically rather than a relic PNe from the hot companion. However, at this time neither the statistical frequency nor the expected lifetime of such a dusty shell as we report here are known, so statistical comparisons of IR shells and optical nebulae are not possible. Based on the available evidence pertaining to $\mathrm{BI} \mathrm{Cru}$, and the present lack of understanding of symbiotic Mira mass outflow histories and possible symbiotic-PNe connections, the origin of the BI Cru IR shell remains uncertain. We are presently undertaking a search for extended IR emission near other symbiotics using Spitzer data in order to obtain better statistics on the frequency of such shells and lobes.

The offset, unipolar nature of the BI Cru IR shell is considerably different from the appearance of any known ionized shell or lobe associated with a symbiotic. Other optical nebulae with symbiotic Miras are aspherical (Corradi et al. 1999b), but have more elongated structures. A fainter region of diffuse, extended emission is visible at $8 \mu \mathrm{m}$ on the northeast side of BI Cru which has a size, shape, and orientation similar to what would be expected from an opposing structure from a bipolar mass outflow. However, the extended emission on the northeast side is clearly unstructured and much fainter, and indeed is not discernible except at $8 \mu \mathrm{m}$ and just possibly at $24 \mu \mathrm{m}$. While it is possible that this feature represents a counterpart of the main shell, it is less convincing because its brightness is similar to that of other (albeit more irregular) patches of ISM emission in the BI Cru area. If it is a counterpart, its relative faintness would still suggest an asymmetric mass ejection. Whether the event leading to the observed IR-shell was initially a single- or double-lobed ejection cannot be answered here. If it were bipolar, then dense material north of the star might have prevented large-scale expansion on that side. In any case, nebulae around D-type symbiotics show a variety of diverse shapes, including rings, ellipses, bent jets, bipolar lobes, and irregular geometries which may represent an equally diverse and complex range of originating processes (Corradi et al. 1999a).

\section{CONCLUSION}

Spitzer IRAC and MIPS images reveal a slightly elongated, shell-like region of dust $\sim 7^{\prime}$ in diameter. We argue that this shell probably resulted from a mass ejection from BI Cru because BI Cru is a known mass ejector; because the known spatial density of such shells $\left(\sim 1.5 \mathrm{deg}^{-2}\right.$ in that region of the Galactic plane) makes it highly improbable that there would be a chance alignment between a shell of that size and a Mira symbiotic; because the shell boundary overlies the position of BI Cru, which is even less probable by chance; and because the shell's major axis, as well as can be estimated, falls along the position angle of the current mass outflow detected optically.

The shell extends more than 5 times farther from the star than does the optical lobe. The location and structure of the IR emission is fairly similar at all wavelengths, except that the 24 and $70 \mu \mathrm{m}$ emission is more diffuse and appears to "fill in" the shell, giving a more lobelike appearance, while at shorter wavelengths emission is concentrated mostly on narrow arcs which mostly form the outer boundary of the shell. The two published distance estimates of BI Cru imply that the shell, if at the same distance, has a physical diameter of $\sim 4$ or $\sim 8 \mathrm{pc}$. There appear to be multiple intersecting arcs in $8 \mu \mathrm{m}$ emission, and to a lesser degree in $5.8 \mu \mathrm{m}$ emission. Although optical mass ejection features are known to exist with a large fraction of D-type symbiotic systems, this is the first extended IR structure to be discovered associated with any symbiotic. The estimated distance of BI Cru implies that the outer edge of the IR lobe is slightly larger than the largest optical lobe known with a symbiotic. The expansion velocity of the IR shell is unknown, but if its velocity is assumed to be the same as smaller optical lobes presently being ejected from BI Cru, then the shell's dynamical age is $\geq 15 \times 10^{3} \mathrm{yr}$, which is $\sim 8$ times longer than the estimated age of the optical lobes. The structure's brightness increases from shorter to longer wavelengths, by a factor of $\sim 10-20$ from 3.6 to $70 \mu \mathrm{m}$, which suggests that there is substantially more cold dust than warm dust present.

This work is based on observations made with the Spitzer Space Telescope, which is operated by the Jet Propulsion Laboratory, California Institute of Technology, under a contract with NASA. This work has used of SAOImage DS9, developed by Smithsonian Astrophysical Observatory; data from the Two Micron All Sky Survey, which is a joint project of the University of Massachusetts and the Infrared Processing and Analysis Center/ California Institute of Technology, funded by the National Aeronautics and Space Administration and the National Science Foundation; and the SIMBAD database, operated at CDS, Strasbourg, France. We thank an anonymous referee for useful comments.

\section{Facilities: Spitzer}

\section{REFERENCES}

Allen, D. A. 1974, Inf. Bull. Variable Stars, 911

Allen, D. A., \& Glass, I. S. 1975, MNRAS, 170, 579

Balick, B. 2003, in ASP Conf. Ser. 303, Symbiotic Stars Probing Stellar Evolution, ed. R. L. M. Corradi, J. Mikolajewska, \& T. J. Mahoney (San Francisco: ASP), 407

Benjamin, R. A., et al. 2003, PASP, 115, 953

Brocksopp, C., Sokoloski, J. L., Kaiser, C., Richards, A. M., Muxlow, T. W. B., \& Seymour, N. 2004, MNRAS, 347, 430

Burgasser, A. J., Kirkpatrick, J. D., McElwain, M. W., Cutri, R. M., \& Skrutskie, M. F. 2003, AJ, 125, 850

Carey, S. J., et al. 2005, BAAS, 37, 1252

Churchwell, E., et al. 2006, ApJ, 649, 759

. 2007, ApJ, 670, 428
Corradi, R. L. M. 2003, in ASP Conf. Ser. 303, Symbiotic Stars Probing Stellar Evolution, ed. R. L. M. Corradi, J. Mikolajewska, \& T. J. Mahoney (San Francisco: ASP), 393

Corradi, R. L. M., Brandi, E., Ferrer, O. E., \& Schwarz, H. E. 1999a, A\&A, 343,841

Corradi, R. L. M., Brandi, E., Ferrer, O. E., Schwarz, H. E., \& García, L. 1999b, A\&A, 348, 978

Corradi, R. L. M., \& Scwharz, H. E. 1993, A\&A, 268, 714

. 1995, A\&A, 293, 871

Finlator, K., et al. 2000, AJ, 120, 2615

Fazio, G. G., et al. 2004, ApJS, 154, 10

Henize, K. G. 1976, ApJS, 30, 491

Henize, K. G., \& Carlson, E. D. 1980, PASP, 92, 479 
Lerche, I. 1980, A\&A, 85, 141

Li, A., \& Draine, B. T. 2002, ApJ, 572, 232

Monet, D. G., et al. 2003, AJ, 125, 984

Morris, P., Stolovy, S., Wachter, S., Noriega-Crespo, A., Pannuti, T. G., \& Hoard, D. W. 2006, ApJ, 640, L179

Neugebauer, G, et al. 1984, ApJ, 278, L1

Reach, W. T., et al. 2006, AJ, 131, 1479

Rieke, G., et al. 2004, ApJS, 154, 25

Rossi, C., Altamore, A., Ferrari-Toniolo, M., Persi, P., \& Viotti, R. 1988, A\&A, 206,279
Schwarz, H. E., \& Corradi, R. L. M. 1992, A\&A, 265, L37

Skrutskie, M. F. 2006, AJ, 131, 1163

Weaver, R., McCray, R., Castor, J., Shapiro, P., \& Moore, R. 1977, ApJ, 218, 377

Wendker, H. J. 1995, A\&AS, 109, 177

Werner, M. W. 2004, ApJS, 154, 1

Whitelock, P. A., Feast, M. W., Roberts, G., Carter, B. S., \& Catchpole, R. M. 1983, MNRAS, 205, 1207

Wright, A. E., Griffith, M. R., Burke, B. F., \& Ekers, R. D. 1994, ApJS, 91, 111 